\title{
Phraseologismen und stereotype Sprechakte im Deutschen und im Französischen
}

\author{
Maurice Kauffer (Nancy)
}

\begin{abstract}
The topic of this paper is the definition and lexicographic treatment of pragmatic phraseologisms and in particular stereotypical speech acts in German and French. We begin with a critical examination of the traditional distinctions within pragmatic phraseologisms, i. e. between formulaic expressions, context-dependent phraseologisms and phraseologisms functioning as sentences. As a result, we propose a new, more clearly delineated set of stereotypical speech acts, i. e. phrases such as Na warte mal!,Sieh mal einer an!, Tu parles ! Tu vois ce que je vois?.Stereotypical speech acts meet three requirements: semantic idiomaticity, utterance value and pragmatic function, and are generally used in spontaneous or fiction dialogues. Finally, we present a context-rich, corpus-based, bilingual dictionary of stereotypical speech acts that is being compiled in Nancy. Content and design of the dictionary are illustrated by two examples, la belle affaire and das ist die Höhe.
\end{abstract}

\section{Kritisches Plädoyer für die pragmatischen Phraseologismen}

\subsection{Klassifizierungsprobleme}

In der phraseologischen Forschung ist die Klassifizierung bzw. Kategorisierung phraseologischer Einheiten von jeher problematisch gewesen. Beim heutigen Forschungsstand sind insbesondere die drei herkömmlichen Definitionskriterien von Phraseologismen, nämlich Polylexikalität (Mehrgliedrigkeit), Festigkeit und Idiomatizität (z. B. bei Burger 1998/2003: 14 oder Greciano 1995: 183) nicht mehr unantastbar. Wer diese Kriterien prinzipiell auf alle Phraseologismen anwenden will, stößt schnell auf erhebliche Schwierigkeiten. Man denke nur an die Kategorie der Festigkeit, deren komplexe Merkmale äußerst schwer zu bewältigen sind (vgl. Stein 2004: 264-265 und Gualberto-Schneider/Kauffer/Nahon-Raimondez (im Druck) und an Schmales erweiterte Definition (2011: 188) "präformierter Konstruktionseinheiten". Stein (1995: 42) verwendet deshalb polare Merkmale (Endpol vs. Anfangspol), um periphere und zentrale phraseologische Einheiten zu unterscheiden.

Im ersten Teil wird erklärt, was wir unter pragmatischen Phraseologismen verstehen. Dieser Terminus ist nicht neu: Burger, Buhofer und Sialm (1982: 41) sprechen schon von "pragmatisch markierten Phraseologismen" und von "pragmatischen Phraseologismen" (dies.:110). Aber der Terminus dient im vorliegenden Aufsatz hauptsächlich zur begrifflichen Herausbildung der stereotypen Sprechakte, auf die wir später eingehen werden. 
Wenn auch manche Bereiche der Phraseologieforschung heutzutage häufiger als andere untersucht werden (idiomatische Wendungen, Sprichwörter), möchten wir uns auf eine andere Klasse von Phraseologismen konzentrieren, nämlich auf pragmatische Phraseologismen. Es sind Phraseologismen, deren Funktion vor allem pragmatischer Natur ist, das heißt - grob gesagt - dass sie eine wichtige Funktion in der sprachlichen Kommunikation und ganz besonders in Gesprächen ${ }^{1}$ haben. Pragmatische Phraseologismen sind besonders interessant nicht nur, weil sie viele Okkurrenzen in den von uns ausgewerteten Korpora gesprochener und geschriebener Sprache haben, sondern auch, weil sie sich noch nicht so sehr der Beliebtheit der Linguisten erfreuen: Verhältnismäßig wenige Aufsätze und Bücher (im Vergleich zu anderen Forschungsbereichen der Phraseologie) haben sich dieser Problematik gewidmet. Dazu sei ebenfalls erwähnt, dass pragmatische Phraseologismen auch in der Fremdsprachendidaktik eine harte Nuss sind: Meine Erfahrung als Dozent für Deutsch als Fremdsprache zeigt, dass Studierende i. d. R. große Schwierigkeiten haben, Phraseologismen dieses Typs zu erkennen, geschweige denn, sich diese anzueignen.

\subsection{Pragmatische Phraseologismen}

Pragmatische Phraseologismen, deren Funktion vor allem kommunikativer Natur ist, finden sich in zwei Klassen von Phraseologismen, nämlich Routineformeln und satzwertigen Phraseologismen. Wir werden feststellen, dass diese Klassen nicht einfach abzugrenzen sind: Nicht alle Routineformeln sind Phraseologismen und Routineformeln können auch satzwertig sein. Hyvärinens Überblick (2011) über die verschiedenen Klassifizierungen pragmatischer Phraseologismen ist zwar überzeugend, aber die Einteilung in (situationsgebundene) Routineformeln und (situationsunabhängige) gesprächsspezifische Formeln - wie sie Stein $(1995,2004)$ auch formuliert hat - erweist sich letzten Endes (ders., 39-41) als nicht hieb- und stichfest. Deswegen bleiben wir vorübergehend bei der Differenzierung Routineformeln vs. satzwertige Phraseologismen.

\subsubsection{Routineformeln}

Nennen wir zuerst einige Beispiele für französische und deutsche Routineformeln:

(1a) pas vrai?/à mon avis/bon rétablissement!

(1b) nicht wahr?/meiner Meinung nach/ gute Besserung!

Die ersten systematischen Untersuchungen der Routineformeln stammen von Gülich, Henke (1979) und Coulmas (1981). Es gibt neuere wichtige Darstellungen u. a. bei Burger (2003: 3637, 53-55), Lüger (2007), Hyvärinen (2011) und Balzer und Piñel (2010). Burger (2003: 36) definiert sie als Phraseologismen, die "bestimmte Aufgaben bei der Herstellung, Definition, dem Vollzug und der Beendigung kommunikativer Handlungen [haben]", aber es gibt in der Forschungsliteratur andere Definitionen der Routineformeln, auf die wir hier nicht näher eingehen können. Besonders interessant sind die Kriterien, die in diesen Definitionen verwendet werden, weil sie, wie wir unten sehen werden, nicht immer klar definiert sind. Welche Kriterien?

\footnotetext{
${ }^{1}$ Gespräch im Sinne von Brinker und Sager (2012: 12), d. h. "eine begrenzte Folge von sprachlichen Äußerungen, die dialogisch ausgerichtet ist und eine thematische Orientierung aufweist".
} 
- Routinecharakter, d. h. die wiederholte Ausführung von (sprachlichen) Handlungen im Diskurs.

- Vorgeformtheit der Routineformeln. Wenn sie aus mehreren Lexemen bestehen, sind sie vorgeformte, sozusagen gebrauchsfertige Wortverbindungen, die nicht bei jedem Gespräch neu gebildet werden müssen.

- Situationsgebundenheit. Fónagy (1997) spricht von énoncé lié ('gebundene Äußerung'), Mel'čuk (2008) von pragmatème. Aber weder Stein (2004) noch Burger (2003) meinen, dass Routineformeln unbedingt situationsgebunden sind. Burger (ders.: 53) unterscheidet "(1) diejenigen, die funktionell an einen bestimmten Situationstyp gebunden sind, [und] (2) diejenigen, die situationsunabhängige kommunikative Funktionen $[\ldots]$ haben".

- Funktion der Routineformeln. Sie besteht hauptsächlich in der Strukturierung des Textes oder des Gesprächs und im "Kommunikationsmanagement" (vgl.Stein 1995).

- Idiomatische Bedeutung. Die Bedeutung der Routineformeln ist im Allgemeinen nicht-kompositionell, d. h. die Gesamtbedeutung ist nicht völlig von den Bedeutungen der Komponenten ableitbar. Aber manche Routineformeln sind nicht-idiomatisch, z. B. nicht wahr? Sobald eine spezifische pragmatische Funktion zur wörtlichen Bedeutung hinzukommt, ist eine Routineformel zumindest teilidiomatisch.

- Satzwertigkeit bei vielen, aber nicht allen Routineformeln: gute Besserung! ist satzwertig, meiner Meinung nach nicht. Wir kommen später auf dieses Kriterium zurück.

Es ist also schwierig, eine einheitliche Begriffsbestimmung der Routineformeln herauszuarbeiten, weil kein Konsens über Kriterien wie Situationsgebundenheit, Vorgeformtheit und Idiomatizität herrscht. Die ständige Änderung der Terminologie in diesem Teilbereich zeigt übrigens, dass die Routineformeln nicht immer den gleichen Stellenwert gehabt haben. Bei Burger z. B. heißen die Routineformeln zuerst "pragmatische Idiome" (1973), dann "pragmatische Phraseologismen" (1982) und schließlich "kommunikative Phraseologismen" und auch Routineformeln" (2003).

\subsubsection{Satzwertige Phraseologismen}

Satzwertige Phraseologismen werden von Burger (2003) "feste Phrasen"2 genannt, bei Lüger (1999) sind sie "satzwertige Phraseologismen". Welche Definitionskriterien ${ }^{3}$ werden in diesen beiden Darstellungen, den wichtigsten in diesem Teilbereich, verwendet? Da kein ausführlicher Forschungsbericht vorgelegt werden kann, gehen wir nicht auf die gesamte Literatur ein; es geht vielmehr um einen Gesamtüberblick über die Definitionskriterien.

\footnotetext{
${ }^{2}$ Burger (2003: 39) differenziert eigentlich zwei Gruppen von "satzwertigen/propositionalen Phraseologismen", nämlich "feste Phrasen" und "topische Formeln". Wir konzentrieren uns auf die erste Gruppe.

${ }^{3}$ Folgende Kriterien beziehen sich nicht auf Burgers (ebd.) "topische Formeln" (d. h. Sprichwörter und Gemeinplätze).
} 
- Satzwertigkeit ist natürlich ein definitorisches Merkmal,

- Vorgeformtheit: Satzwertige Phraseologismen sind, wie alle Phraseologismen, griffbereite Wortverbindungen, die nicht jedes Mal neu gebildet werden müssen,

- Kontextgebundenheit: Sie sind an den Kontext angeschlossen, meistens durch exophorische oder anaphorische Pronomina (vgl. Burger 1998/2003: 39),

- Idiomatizität, d. h. idiomatische Bedeutung,

- pragmatische Funktion. Z. B. gibt in das ist die Höhe! der Sprecher zu verstehen, dass etwas zu weit geht. Bei voyez-vousça! zweifelt der Sprecher an etwas.

Es stellt sich in 1.2.1 und 1.2.2 heraus, dass die oben erwähnten Kriterien weder trennscharf noch eindeutig sind, nämlich aus drei Gründen.

Es wurde oben darauf hingewiesen, dass pragmatische Phraseologismen nicht immer situationsabhängig sind. Manchmal tauchen sie zwar nur in einer bestimmten Kommunikationssituation auf. Ich sage z. B. Gute Besserung! nur, wenn ich meinem Kommunikationspartner, der krank ist, wünsche, dass er schnell wieder gesund wird. Aber bei einigen Phraseologismen wie z. B. nicht wahr? oder Sieh mal einer an! ist das nicht der Fall: Sie können in vielen verschiedenen Situationen gebraucht werden. Eine Differenzierung unterschiedlicher Typen von situationsspezifischen Phraseologismen wäre notwendig, würde jedoch den Rahmen unserer Darstellung sprengen.

Man kann sich fragen, ob Satzwertigkeit ein stichhaltiges Merkmal ist. Viele pragmatische Phraseologismen bestehen nicht aus einem Satz mit Verb, z. B. Gute Besserung!; Und wie!; oder Ach was!, aber sie bilden doch eine Äußerung. Eine Äußerung ist nicht unbedingt ein Satz mit einem Verb, z. B. Schluss! oder na und!. Zifonun, Hoffmann und Strecker (1997: 86) unterscheiden auch Satz und Äußerung bzw. "kommunikative Minimaleinheit". Nicht die Satzwertigkeit, sondern die "Äußerungswertigkeit" spielt also die entscheidende Rolle bei den "pragmatischen Phraseologismen".

Auf pragmatische Funktionen wird im weiteren Verlauf des Beitrages einzugehen sein.

Es ergibt sich letzten Endes, dass Situationsabhängigkeit, Äußerungswertigkeit und pragmatische Funktion wichtige - aber auch problematische - Definitionskriterien eines pragmatischen Phraseologismus sind. Diese kommunikationszentrierten Kriterien stehen im Mittelpunkt einer neuen, besser abgegrenzten Klasse der stereotypen Sprechakte, auf die wir jetzt eingehen.

\section{Stereotype Sprechakte}

\subsection{Begriffsbestimmung}

Wir definieren stereotype Sprechakte bzw. StSa (auf Französisch ALS, d. h. "actes de langage stéréotypés") als Wendungen, die drei Voraussetzungen erfüllen müssen. 


\subsubsection{Erste Voraussetzung: Idiomatizität}

Der StSa hat eine nicht-kompositionelle Bedeutung: Seine Bedeutung kann nicht vollständig von der Bedeutung der Komponenten der Wortverbindung abgeleitet werden. Die deutschen und französischen Beispiele in (2a) sind StSa mit idiomatischer Bedeutung, die in (2b) sind keine StSa, weil sie nicht idiomatisch sind.

(2a) na warte, Freundchen! ('eine Warnung wird ausgedrückt')

allons donc!

('der Sprecher bezweifelt etwas')

c'est le bouquet!

$(\approx \text { 'das ist der Gipfel' })^{4}$

(2b) warte (auf meinen Anruf)!

allons donc (au café)!

c'est un bouquet!

Dabei spielt der Kontext eine besonders große Rolle, nicht nur hinsichtlich der Bedeutung der StSa, sondern auch, was ihre Zuordnung zu der Klasse der StSa angeht. Sehr ähnliche oder sogar identische Wendungen können je nach Kontext zu den StSa gehören oder nicht, weil sie idiomatisch (3a) oder nicht idiomatisch (3b) sind:

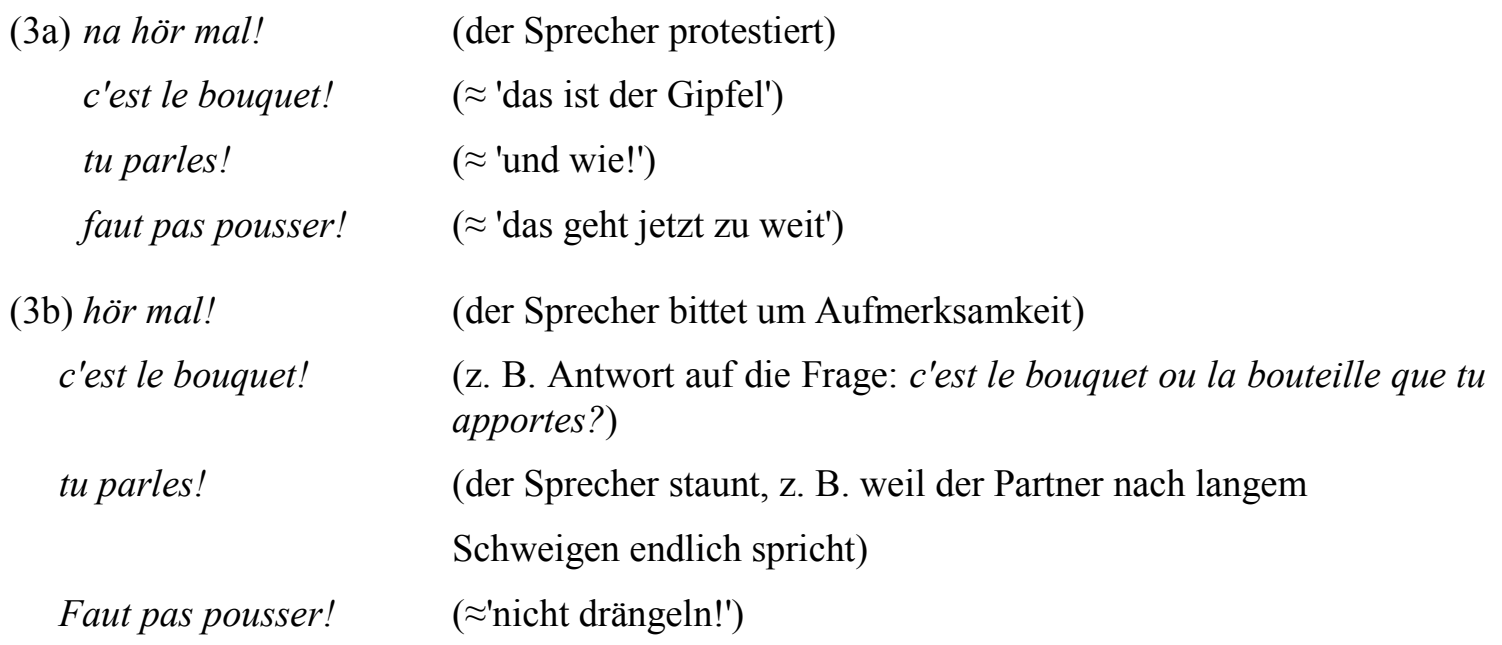

\subsubsection{Zweite Voraussetzung: Äußerungswertigkeit}

Ein StSa muss immer eine autonome Äußerung sein können. Na warte! und la belle affaire sind also Äußerungen in (4a), aber nicht in (4b):

Na warte! Ich werde dir lächeln helfen!

"Si je ne chasse pas, Madame, j'ai des vapeurs.

La belle affaire! J'en ai bien, moi, et je n'en mourrai pas" (Chandernagor 1981: 603)

Na, warte, bis dein Vater das erfährt!

La belle affaire qu'il a faite en achetant cette voiture.

\footnotetext{
${ }^{4}$ Das Zeichen $\approx$ steht in diesem Aufsatz nicht für Identität, sondern für pragmatisch-semantische Äquivalenz.
} 


\subsubsection{Dritte Voraussetzung: pragmatische Funktion}

Der StSa hat zwar eine Referenzfunktion, aber hauptsächlich eine pragmatische Funktion. Durch den StSa wird ein Sprechakt vollzogen. Der StSa hat also eine illokutionäre Kraft. In der Regel drückt der StSa eine Reaktion des Sprechers auf eine Aussage oder auf einen Sachverhalt aus. Deswegen kommen stereotype Sprechakte sehr oft in Gesprächen bzw. in Dialogen vor.

Stereotype Sprechakte können verschiedene pragmatische Funktionen haben. Führen wir ein paar Beispiele an:

- Ausdruck von Staunen: sieh mal (einer) an!/tu vois ce que je vois?/eh ben dis donc!

- Warnung, Drohung: na warte!/vous allez voir ce que vous allez voir!

- Ablehnung (sehr häufige Funktion): rutsch mir den Buckel runter!/steig mir den Buckel rauf!/ tu peux toujours courir!/compte là-dessus!

- Ärger, Empörung: das ist die Höhe!/du kannst mich mal/ça va pas la tête?

Pragmatische Funktionenkönnen auch je nach Kontext variieren. Zum Beispiel bekundet $t u$ parles! folgende, sehr unterschiedliche Reaktionen des Sprechers:

- etwas abstreiten, verneinen ( $\approx$ 'denkste!'/ 'ach was!')

(5a) "Tout ce qu'il gagne, il le met dans son entreprise"

"Tu parles! Je suis sûre qu'il te couvre d'or." (Buron 1996: 135)

- etwas bekräftigen ( $\approx$ 'und wie!')

(5b) "T'as eu les jetons?

Tu parles. Jamais eu une telle trouille de ma vie." (Queneau 1967: 49)

Aber tu parles ist bei weitem kein Einzelbeispiel. Deswegen ist es notwendig, eine Bestandsaufnahme allerkontextuellen Funktionen eines $\mathrm{StSa} \mathrm{zu}$ machen und sie $\mathrm{zu}$ klassifizieren.

\subsubsection{Analysen stereotyper Sprechakte}

In der einschlägigen Literatur gibt es im Grunde genommen keine Analysen stereotyper Sprechakte, die diese Klasse als solche definieren und sie von anderen Klassen abgrenzen. Aber einige Phraseologen interessieren sich dennoch für die Beziehung zwischen Phraseologismen und Sprechakten. Franz Schneider (1989) definiert den Begriff "expression d'illocutoire stéréotypé" (EIS) im Französischen. Aber dieser Begriff ist sehr weit gefasst: Schneider meint, dass alle Wendungen EIS sind, wenn sie nur imstande sind, eine illokutionäre Kraft zu haben. So gehören sowohl tu parles! als auch parlons-en! und parlez-moi de lui! zu den EIS. Die EIS sind übrigens nicht immer idiomatisch, und auch nicht äußerungswertig. Schemann (1993) führt den sehr interessanten Terminus "lexikalisierte Sprechakte" ein und behauptet, dass die "lexikalisierten Sprechakte" sprechaktgebunden sind, d. h., dass jeder nur von einem Sprechakt abhängt, was nicht immer stimmt (siehe tu parles!). Und er erwähnt auch nicht die Äußerungswertigkeit der lexikalisierten Sprechakte. Françoise Bidaud (2002) untersucht die "structures figées de la conversation", nämlich Wendungen, die festgeprägt sind, zwei 
Bedeutungen je nach Kontext haben, einen geringen Grad an Idiomatizität aufweisen und auch eine Reaktion auf einen gewissen Stimulus bilden. Andere Analysen finden sich in Aufsätzen von Martins-Baltar (2006, 2000), Dostie (2004), Dziadkiewicz (2007) und Lamiroy (2010).

\subsection{Zur Festigkeit der "stereotypen Sprechakte"}

\subsubsection{Morpho-syntaktische Festigkeit}

Diese Festigkeit besteht aus einer Reihe von Restriktionen und Irregularitäten (vgl. Burger 1998/2003: 20-22). Die morpho-syntaktische Festigkeit von StSa ist i. A. ziemlich stark, obwohl sie eine graduelle Eigenschaft darstellt und verschiedene Grade der Festigkeit existieren können. Mögliche Restriktionen und Irregularitäten sind zum Beispiel:

- das eingeschränkte Variieren von Tempus, Modus, Numerus, Person usw. Z. B. steht na warte! als StSa nur im Imperativ,

- der prädikative (oder attributive) Gebrauch des Adjektivs ist unmöglich: als StSa ist la belle affaire möglich, aber nicht *l'affaire est belle,

- kein Hinzufügen von (z. B. adjektivischen) Attributen: das ist die Höhe!, aber nicht *das ist die große Höhe!,

- $\quad$ keine paradigmatische Variation des StSa: la belle affaire, aber nicht *la jolie affaire.

\subsubsection{Semantische Festigkeit (= Idiomatizität)}

Die StSa haben i. d. R. zwei Bedeutungen, eine wörtliche und eine phraseologische. Bei manchen StSa ist jedoch keine wörtliche Bedeutung möglich, d. h. der StSa besitzt nur eine idiomatische Bedeutung, z. B. Von wegen/ Pustekuchen!/ tant qu'à faire ( $\approx$ 'wennschon, dennschon'). Die Idiomatizität kann schwach sein: hast du Worte!/ voyez-vous ça! ('sieh mal einer an!'), oder auch stark: rutsch' mir den Buckel runter!/ Schwamm drüber/ c'est le bouquet ( $\sim^{\prime}$ das ist die Höhe')/à d'autres! (Ausdruck der Ungläubigkeit).

\subsubsection{Pragmatische Festigkeit}

Auf dieser Ebene sollten zwei Aspekte berücksichtigt werden. Einerseits die mehr oder weniger enge Beziehung zwischen dem StSa und seiner illokutionären Kraft (Ablehnung, Warnung, Zustimmung usw.). Andererseits die Tatsache, dass diese illokutionäre Kraft mit deiktischen Pronomina und Adverbien verbunden ist: da lachen ja die Hühner/ jetzt ist Sense/ voyez-vous ça! ( $\approx$ 'sieh mal einer an!') (vgl. Gualberto-Schneider/Kauffer/Nahon-Raimondez (im Druck)).

\subsection{StSa und Sprechakte}

In der folgenden Tabelle werden einige deutsche und französische Beispiele von Tokens StSa mit den entsprechenden Sprechakt-Typen präsentiert. 


\begin{tabular}{|c|c|}
\hline Sprechakt & $\mathrm{StSa}$ \\
\hline $\begin{array}{l}\text { jm. zustimmen, } \\
\text { ein positives Urteil } \\
\text { abgeben }\end{array}$ & $\begin{array}{l}\text { - du sagst es!/das lässt sich hören/klarer Fall! } \\
\text { - c'est du billard/c'est-y pas beau, ça?/j'en suis fort aisel je n'y } \\
\text { manquerai pas/l'affaire est dans le sac/tu l'as dit (bouffi)! }\end{array}$ \\
\hline $\begin{array}{l}\text { eine Aussage } \\
\text { bekräftigen, } \\
\text { bestätigen }\end{array}$ & $\begin{array}{l}\text { - das kann man wohl sagen!/ist gebongt/na also!/und wie! } \\
\text { - c'est le cas de le dire/c'est le moins qu'on puisse dire/ça m'en a } \\
\text { tout l'air/ et comment!/et pour cause je ne connais que ça/j'en } \\
\text { passe et des meilleures }\end{array}$ \\
\hline $\begin{array}{l}\text { eine Aussage oder } \\
\text { einen Fakt banali- } \\
\text { sieren, relativieren }\end{array}$ & $\begin{array}{l}\text { - c'est bonnet blanc et blanc bonnet/c'est de la petite bièrel ce } \\
\text { n'est pas la mer à boire!/la belle affaire! }\end{array}$ \\
\hline $\begin{array}{l}\text { etw. mildern, } \\
\text { dämpfen }\end{array}$ & $\begin{array}{l}\text { - immer mit der Ruhe!/sachte, sachte! } \\
\text { - calmos!/comme tu y vas!/doucement les basses/ mollo/ n'en jetez } \\
\text { plus! }\end{array}$ \\
\hline $\begin{array}{l}\text { kritisieren, ein } \\
\text { negatives Urteil } \\
\text { abgeben }\end{array}$ & $\begin{array}{l}\text { - aber, aber/Fehlanzeige! } \\
\text { - beaucoup de bruit pour rien/ça ne casse pas trois pattes à un } \\
\text { canard/tu en as de bonnes/tout de même! }\end{array}$ \\
\hline $\begin{array}{l}\text { jn. warnen } \\
\text { jm. drohen }\end{array}$ & $\begin{array}{l}\text { - mein lieber Freund und Kupferstecher/na warte (Freundchen)! } \\
\text { - attachez vos ceintures/essaie voir/tu vas voir ce que tu vas voir!/ } \\
\text { viens-y!/tu ne perds rien pour attendre }\end{array}$ \\
\hline $\begin{array}{l}\text { etw. ablehnen, } \\
\text { zurückweisen }\end{array}$ & $\begin{array}{l}\text { - ach was!/ach wo!/denkste!/Pustekuchen/rutsch mir den Buckel } \\
\text { runter!/steig mir den Buckel rauf! } \\
\text { - allons donc!/compte là-dessus/des clous!/de l'air!/du balai!/du } \\
\text { vent!/en voilà assez!/tu peux toujours courir! }\end{array}$ \\
\hline mit etw. abschließen & $\begin{array}{l}\text { - der Bart ist ab/Schwamm drüber!/und damit basta! } \\
\text { - affaire classée!/accordez vos violons!/basta! c'est tout vu/et à } \\
\text { part ça? }\end{array}$ \\
\hline $\begin{array}{l}\text { Eine negative Bilanz } \\
\text { ziehen }\end{array}$ & $\begin{array}{l}\text { - Pech gehabt!/den Letzten beißen die Hunde } \\
\text { - c'est là que les Athéniens s'atteignirent/nous voilà bien!/pas de } \\
\text { bol }\end{array}$ \\
\hline staunen & $\begin{array}{l}\text { - ach was/au Backe/du bist vielleicht gut!/meine Güte/Mensch } \\
\text { Meier/mich laust der Affe/sieh mal einer an! } \\
\text { - eh ben dis donc!/tu vois ce que je vois? }\end{array}$ \\
\hline resignieren & $\begin{array}{l}\text { - sei's drum!/was solls? } \\
\text { - c'est comme ça/les carottes sont cuites/sans commentaire }\end{array}$ \\
\hline
\end{tabular}




\begin{tabular}{|l|l|}
\hline sich empören & $\begin{array}{l}\text { - das ist die Höhe!/das ist der Gipfel/du kannst mich mal/dich } \\
\text { hat's wohl!/was soll das? } \\
\\
\text {-ça va pas la tête?/c'est le comble/c'est le bouquet }\end{array}$ \\
\hline
\end{tabular}

Tabelle 1:StSa und Sprechakte

\section{Stereotype Sprechakte und Lexikographie}

In diesem dritten Teil wird das lexikographische Projekt präsentiert, das zurzeit in Nancy durchgeführt wird. Es geht um die Erstellung eines neuen phraseologischen Wörterbuchs der stereotypen Sprechakte.

\subsection{Organisatorischer Rahmen}

Die Teilnehmer am lexikographischen StSa-Projekt unter meiner Leitung sind sieben Germanisten und ein Anglist der Universität Nancy (Maurice Kauffer, Anja Smith, René Métrich, Anne-Marie Nahon-Raimondez), der Universität Metz (Yvon Keromnès, Caroline Pernot) und der Universität Straßburg (Vincent Balnat, Antje Gualberto-Schneider). Es besteht auch eine aktive Kooperation mit Romanisten der Universität Bayreuth (Martina Drescher, Katrin Pfadenhauer).

Das Forschungsteam GLFA (Groupe de lexicographie franco-allemande ${ }^{5}$ ) ist Bestandteil des ATILF, eines CNRS-Forschungslabors im Bereich Französische Sprache und Informatik in Nancy. Folgende Publikationen zeigen die Erfahrung des GLFA auf dem Gebiet der deutschfranzösischen Lexikographie:

- Les Invariables difficiles. Dictionnaire allemand-français des particules, connecteurs, interjections et autres 'mots de la communication' (1992-2002), 4 Bände, Nancy.

- Lexitec (2005): elektronisches zweisprachiges Phraseologie-Wörterbuch, das in die Übersetzungssoftware Reverso integriert ist.

- Wörterbuch deutscher Partikeln (2010).

- Dictionnaire français-allemand des mots du discours: französisch-deutsches Pendant der Invariables difficiles, an dem unser Kollege René Métrich zurzeit arbeitet.

\subsection{Das geplante Wörterbuch}

Das Wörterbuch hat folgende charakteristische Merkmale. Es ist ein zweisprachiges Wörterbuch (Französisch-Deutsch, Deutsch-Französisch ${ }^{6}$ ) der stereotypen Sprechakte. Auf einer vorläufigen Liste der StSa stehen etwa 900 Kandidaten: 470 französische und 430 deutsche stereotype Sprechakte.

Es hätte ein onomasiologisch angelegtes Wörterbuch mit thematischer Gruppierung je nach Sprechakten sein können. In diesem Fall würde aber der Zugang zu den Artikeln nur über einen

\footnotetext{
${ }^{5}$ Siehe http://www.atilf.fr/spip.php?rubrique203, Stand: 19.11.2013.

${ }^{6}$ Das Wörterbuch ist noch im Anfangsstadium, deshalb wird gleichzeitig in beiden Richtungen gearbeitet. Es sollen später zwei Wörterbücher, Deutsch-Französisch und Französisch-Deutsch, entstehen.
} 
Index erfolgen. Wir haben uns also für ein semasiologisches Wörterbuch mit alphabetischer Anordnung des Wörterverzeichnisses $-\mathrm{d}$. h. der StSa-entschieden. ${ }^{7}$

Es ist in den ersten beiden Teilen des vorliegenden Aufsatzes festgestellt worden, dass jeder StSa unterschiedliche Bedeutungen und Funktionen im Kontext, d. h. in den Texten, in denen er gebraucht wird, aufweist. Deshalb ist es von großem Interesse, dass einerseits der Kontext der StSa und anderseits der Kontext der Übersetzungen der StSa im Wörterbuch stehen. Also wird dieses StSa-Wörterbuch auch ein kontextuelles Wörterbuch sein, insofern als relevante Belege jeder Bedeutung eines StSa im Wörterbuchartikel stehen.

Es soll auch ein korpusbasiertes Wörterbuch sein. Möglichst viele Belege sind erforderlich, um nicht nur die häufigsten, sondern alle kontextuellen Verwendungen darzustellen. Einsprachige und zweisprachige Korpora sind also ein Muss, um der Vielfalt der kontextuellen Bedeutungen gerecht zu werden.

Ein Wörterbuch hat auch Benutzer und soll sie zufriedenstellen. Dieses Wörterbuch sollte sowohl Linguisten, nämlich Romanisten und Germanisten (auch in den Bereichen Deutsch als Fremdsprache und Französisch als Fremdsprache), als auch Übersetzern von Nutzen sein. Das Ziel des Wörterbuchs besteht also darin, einen Thesaurus von allen Bedeutungen und Verwendungen der StSa in beiden Sprachen zu erstellen. Insofern ist es etwas Neues: Es gibt zwar schon viele zweisprachige Phraseologismen-Wörterbücher, aber pragmatische Phraseologismen und besonders stereotype Sprechakte sind bis jetzt von den Lexikographen stiefmütterlich behandelt worden. Die Klasse der StSa wurde im Grunde genommen bis jetzt noch nicht als solche analysiert, weder von Germanisten noch von Romanisten, und wurde auch noch nicht lexikographisch erfasst.

\subsection{Quellen und Korpora}

Wie wird das Wörterbuch erstellt? Der erste Schritt ist die Suche nach den StSa, um die Makrostruktur des Wörterbuchs auszuarbeiten. Der zweite besteht dann in der Suche nach Belegen, d. h. nach Verwendungen der StSa in beiden Sprachen.

\subsubsection{Erster Schritt: Suche nach den StSa}

Automatische Recherchen nach den StSa in Korpora sind sehr schwierig, weil jeder StSa drei definitorische Voraussetzungen erfüllen muss (vgl. 2.1). Viele - aber bei weitem nicht alle StSa sind also in einsprachigen, entweder allgemeinen oder phraseologischen Wörterbüchern zu finden. I. d. R. stehen sie aber meistens ohne Beispiele und ohne präzise Angaben zu ihrer Bedeutung und ihrer pragmatischen Funktion. Die ertragreichsten Wörterbücher sind:

- deutsche Wörterbücher: Deutsches Universalwörterbuch (Duden 2003), Redewendungen und sprichwörtliche Redensarten (Duden 11 2002), Deutsche Idiomatik: Die deutschen Redewendungen im Kontext (Schemann1993), Digitales Wörterbuch der deutschen Sprache (dwds.de, Stand: 31.10.2013).

- französische Wörterbücher: Le Petit Robert, Larousse de la Langue Française, Littré, Trésor de la langue français informatisé (= TLFi, Online-Wörterbuch mit freiem

\footnotetext{
${ }^{7}$ Es gibt natürlich andere Gründe für oder gegen diese Anordnung, die hier nicht i. E. dargestellt werden können.
} 
Zugang, in Nancy erstellt: http://atilf.atilf.fr, Stand: 31.10.2013), Dictionnaire des expressions et locutions (Rey/Chantreau 1997).

Auch bestimmte zweisprachige Wörterbücher können von Nutzen sein; sowohl allgemeine (Pons, Larousse, Harrap's) als auch phraseologische Wörterbücher (Bárdosi/Ettinger/Stölting 2003, Coulon-Mrosowski 1992, Gspann 1971, Hullmann 1996, Kauffer 2007, Schulz/Griesbach 1975, Werny/Snyckers 1982, Zimmer 1990). Aufschlussreiche Bemerkungen zur zweisprachigen Lexikographie finden sich bei Dobrovolski (1999), HigiWydler (1989), Korhonen (2007), Kühn (1983), Lubensky/Mac Shane (2007) und Müller/Kunkel-Razum (2007).

\subsubsection{Zweiter Schritt: Suche nach Belegen in Texten}

Die Suche erfolgt in verschiedenen Korpora geschriebener Sprache:

- Korpora von Bitexten, d. h. französischen Texten mit deutscher Übersetzung oder umgekehrt. Sehr hilfreich sind das GLFA-Korpus, das in Nancy angelegt wurde (etwa 400 literarische Bitexte), und einige deutsch-französische Zeitschriften: Rencontres (rencontres.de, Stand: 31.10.2013), Deutschland (magazine-deutschland.de, Stand: 31.10.2013).

- einsprachige Korpora: Mehrere französische elektronische Textkorpora sind in Nancy (ATILF) erstellt worden: Frantext (mehr als 4000 Werke: frantext.fr, Stand: 31.10.2013), die phraseologische Datenbank BPFQ (Base de phraséologie $d u$ françaisquotidien), bald on line, L'Est Républicain (lothringische Tageszeitung: cnrtl.fr/corpus/estrepublicain, Stand: 31.10.2013). Manche E-Books sind auch nützlich.

Die wichtigsten deutschen Textkorpora sind das Korpus des Instituts für deutsche Sprache in Mannheim (ids-mannheim.de, Stand: 31.10.2013), das Wortschatz-Portal der Universität Leipzig (http://wortschatz-uni.leipzig.de, Stand: 31.10.2013), Zeitungskorpora (DVDs und das Online-Archiv der NZZ, der $S Z$, der Zeit usw.), die Literatur-DVD Gü-DE (11. Auflage) und auch E-Books.

\subsection{Schwierigkeiten}

Das schwierigste Problem hinsichtlich der Erstellung des StSa-Wörterbuchs besteht in der Auswahl der StSa. Wenn StSa-Kandidaten gewählt werden sollen, ist die Entscheidung nicht immer einfach, sie hat oft mit der problematischen Abgrenzung der StSa von anderen Klassen zu tun. Folgende Probleme machen unserem Team zu schaffen:

Die Idiomatizität der StSa ist manchmal schwach. Das führt dazu, dass die wörtliche Bedeutung von der phraseologischen schwer zu unterscheiden ist, was grundsätzlich bei allen Phraseologismen der Fall sein kann. Zum Beispiel: das kann man wohlsagen! oder damit ist alles gesagt. Der StSa je vois bedeutet sogar je nach Kontext entweder 'ich sehe' oder 'ich verstehe' oder auch 'ich bezweifle etwas'. Trotzdem sollen diese StSa auch untersucht werden, weil sie oft gebraucht werden und viele Verwendungen aufweisen.

Polylexikalitätist auch ein schwieriger Punkt. Manche StSa bestehen aus einem einzigen Wort. Es sind zweifellos StSa, aber nicht unbedingt Phraseologismen, die im Prinzip mehrgliedrig sind. Zum Beispiel: Fehlanzeige!;Pustekuchen!; taratata! (bekundet Skepsis, Zweifel); tintin! 
('du wirst nichts bekommen'). Bei den beiden letzten StSa stellt sich sogar heraus, dass die Grenze zwischen StSa und Interjektionen recht fließend ist. Deshalb werden bei diesem lexikographischen Projekt alle StSa akzeptiert, die die drei Grundvoraussetzungen erfüllen (vgl. 2.1), obwohl sie keine Phraseologismen im engeren Sinne sind, wenn sie aus einem Wort bestehen.

Es stehen uns zwar viele umfangreiche Korpora zur Verfügung, trotzdem reichen sie nicht immer aus. Um die Verwendungen von jedem StSa einzusammeln und zu strukturieren, soll uns eine große Anzahl von Belegen zur Verfügung stehen, d. h. wenigstens fünfzig pro StSa. Deswegen soll in Zukunft das GLFA-Korpus in Nancy erweitert werden, andere Quellen müssen herangezogen werden. Bis jetzt wurden in diesem Projekt nur ausnahmsweise Korpora gesprochener Sprache ausgewertet, weil sie noch relativ selten sind und noch nicht umfangreich genug, um ausreichend Okkurrenzen zu liefern.

\subsection{Inhaltsprogramm des Wörterbuchs}

Welche lexikographischen Informationen sollen ins StSa-Wörterbuch aufgenommen werden und in jedem Wörterbuchartikel stehen(s. auch Métrich 2011 dazu)? Um eine erste Antwort auf diese Frage $\mathrm{zu}$ geben, werden diese Inhalte anhand von zwei Beispielen exemplarisch dargestellt, la belle affaire und das ist die Höhe. Es handelt sich jedoch nicht um eine ausführliche Analyse: Nur die Informationen, die künftig im Wörterbuchartikel stehen, werden hier in Form von einigen Tabellen knapp präsentiert.

\subsubsection{Morphologie des StSa}

Die wichtigsten morphologischen Merkmale der StSa la belle affaire und das ist die Höhe stehen in Tabelle 2: eventuelle Varianten, Betonung des StSa und schließlich morphosyntaktischen Restriktionen, die die morpho-syntaktische Festigkeit des StSa ausmachen.

\begin{tabular}{|c|c|c|}
\hline & la belle affaire & das ist die Höhe \\
\hline Phrase & $\begin{array}{l}\text { la belle affaire ist eine } \\
\text { Nominalphrase }\end{array}$ & das ist die Höhe ist ein Satz \\
\hline Varianten & keine & es ist die Höhe (selten) \\
\hline Phonetik & $\begin{array}{l}\text { starke Betonung von belle } \\
\text { möglich }\end{array}$ & $\begin{array}{l}\text { Betonung von Höhe oder von } \\
\text { das (wenn das Pronomen } \\
\text { anaphorisch gebraucht wird) }\end{array}$ \\
\hline $\begin{array}{l}\text { Morpho- } \\
\text { syntaktische } \\
\text { Festigkeit }\end{array}$ & $\begin{array}{l}\text { - StSa nur im Singular, nicht } \\
\text { im Plural } \\
\text { - StSa nur mit bestimmtem } \\
\text { Artikel } \\
\text { - Adjektiv belle nur als } \\
\text { Attribut (kein prädikativer } \\
\text { Gebrauch) }\end{array}$ & $\begin{array}{l}\text { - Tempus: Präsens und } \\
\text { Präteritum sind möglich, aber } \\
\text { zusammengesetzte Formen } \\
\text { nicht (Perfekt, } \\
\text { Plusquamperfekt). } \\
\text { - Modus: Belege im } \\
\text { Indikativ, im Konjunktiv I und } \\
\text { II. }\end{array}$ \\
\hline
\end{tabular}




\begin{tabular}{|l|l|l|}
\hline & - keine paradigmatische & \\
Variation (weder ein anderes & \\
Adjektiv, noch ein anderes & Substantiv) & \\
\hline
\end{tabular}

Tabelle 2: Morpho-syntaktische Merkmale des StSa

\subsubsection{Syntaktische Einbettung des StSa}

Die wichtigsten Kriterien sind die Stellung des StSa im Satz und der syntaktische Status des StSa. Der StSa kann ein Satz bzw. eine autonome Äußerung oder ein Satzglied oder ein Gliedteil sein. In der nächsten Tabelle stehen nur die StSa, wenn sie syntaktisch eingebettet, d. h. a priori nicht äußerungswertig sind. Im Grunde können sie jedoch durch äußerungswertige StSa paraphrasiert werden: la belle affaire qu'un teint fleuri! hat die gleiche Bedeutung wie un teint fleuri: la belle affaire! (erste Zeile von Tabelle 3).

\begin{tabular}{|c|c|c|}
\hline & la belle affaire & das ist die Höhe \\
\hline $\begin{array}{l}\mathrm{StSa}+ \\
\mathrm{NP}^{8}\end{array}$ & $\begin{array}{l}\text { la belle affaire que }+\mathrm{NP} \\
\text { la belle affaire qu'un teint } \\
\text { fleuri!(Sartre 1943: 43) }\end{array}$ & $\begin{array}{l}\text { das ist die Höhe+ NP } \\
\text { Das ist die Höhe des } \\
\text { unschuldigen Nichtwissens (...) } \\
\text { (Donaukurier.de 2008) }\end{array}$ \\
\hline $\begin{array}{l}\mathrm{StSa}+ \\
\text { Nebensatz }\end{array}$ & $\begin{array}{l}\text { la belle affaire que... } \\
\text { la belle affaire si... }\end{array}$ & $\begin{array}{l}\frac{\text { das ist die Höhe }+ \text { dass-Satz }}{\text { das ist die Höhe }+ \text { wenn-Satz }} \\
\text { Es wäre die Höhe, wenn die St. } \\
\text { Geörgler ablehnen }[\ldots] \\
\text { würden. (St. Galler } \\
\text { Tagblatt1999, IdS-Korpus) }\end{array}$ \\
\hline $\begin{array}{l}\mathrm{StSa}+ \\
\text { Infinitiv- } \\
\text { phrase }\end{array}$ & $\begin{array}{l}\text { la belle affaire (que) de }+ \text { Infinitiv- } \\
\text { phrase } \\
\text { La belle affaire de ne pas convoiter } \\
\text { le boeuf du prochain! (Cohen 1930: } \\
\text { 334) }\end{array}$ & $\begin{array}{l}\text { das ist die Höhe +Infinitiv- } \\
\text { phrase (mit zu) } \\
\text { Es ist ja wohl die Höhe, von } \\
\text { seiner Bank rationiert zu } \\
\text { werden!(Dorner 2008: 116) }\end{array}$ \\
\hline $\begin{array}{l}\text { StSa }+ \\
\text { Präposi- } \\
\text { tional- } \\
\text { phrase }\end{array}$ & $\begin{array}{l}\text { la belle affaire + Präpositionalphr. } \\
\text { Ses grands-parents s'en vont? La } \\
\text { belle affaire pour lui. Il ne les } \\
\text { connaît pas...(Ikor 1955: } 308 \text { ) }\end{array}$ & Kein Beleg \\
\hline
\end{tabular}

Tabelle 3: Syntaktische Einbettung desStSa

${ }^{8} \mathrm{NP}$ steht für Nominalphrase. 


\subsubsection{Semantik des StSa}

Tabelle 4 zeigt, dass der StSa eine wörtliche Bedeutung besitzen kann, dass er zu anderen Wendungen in der Relation der Bedeutungsgleichheit bzw. Bedeutungsähnlichkeit stehen kann, und dass ähnliche StSa nach einem bestimmten Modell gebildet werden können.

\begin{tabular}{|c|c|c|}
\hline & la belle affaire & das ist die Höhe \\
\hline $\begin{array}{l}\text { Semantische } \\
\text { Festigkeit }\end{array}$ & $\begin{array}{l}\text { wörtliche Bedeutung möglich } \\
\text { (aber selten) }\end{array}$ & $\begin{array}{l}\text { wörtliche Bedeutung möglich } \\
\text { [...] das ist die Höhe der Beute des } \\
\text { Überfalls auf der Westbahnstrecke } \\
\text { [...] (Die Presse1994, IdS-Korpus) }\end{array}$ \\
\hline $\begin{array}{l}\text { Synonyme/ } \\
\text { Konkurrenten } \\
\text { des StSa }\end{array}$ & $\begin{array}{l}\text { et (puis) après? } \\
\text { et (puis) alors? } \\
\text { peu importe } \\
\text { qu'importe }\end{array}$ & $\begin{array}{l}\text { da hört (sich) doch (einfach) alles } \\
\text { auf/das geht zu weit/das geht über } \\
\text { die Hutschnur/das geht auf keine } \\
\text { Kuhhaut/das ist ein starkes Stück/das } \\
\text { ist allerhand/das ist der Gipfel/das } \\
\text { ist empörend/das ist kaum zu } \\
\text { überbieten/das ist starker Tobak/das } \\
\text { ist unglaublich/das ist unerhört/das } \\
\text { ist unverschämt/das ist eine } \\
\text { Unverschämtheit/das setzt doch } \\
\text { allem die Krone auf/das ist der } \\
\text { Gipfel der Unverschämtheit/so was } \\
\text { von Unverschämtheit! }\end{array}$ \\
\hline $\begin{array}{l}\text { Bildungsmodell } \\
\text { von ähnlichen } \\
\text { StSa }\end{array}$ & $\begin{array}{l}\text { NP vom Typ la belle } N / \text { le } \\
\text { beau } N: \text { mit } \\
\text { "antiphrastischer"9 } \\
\text { Bedeutung: } \\
\text { la belle excuse! ( } \approx \text { 'schöne } \\
\text { Ausrede!') } \\
\text { la belle équipe! ( } \approx \text { 'das sind } \\
\text { mir vielleicht welche!') }\end{array}$ & I \\
\hline
\end{tabular}

Tabelle 4: Semantik des StSa

\subsubsection{Gebrauch des StSa}

Die fünfte Tabelle zeigt einige wichtige Merkmale, die den Gebrauch des StSa ausmachen, insbesondere die Sprachschicht und die häufigen Partner des StSa im Diskurs.

\footnotetext{
${ }^{9}$ Antiphrasis im Sinne von Glück (1993: 43), d. h. "rhetor. Stilmittel, Tropus: meint das Gegenteil des Gesagten".
} 


\begin{tabular}{|l|l|l|}
\hline Gebrauch & la belle affaire & das ist die Höhe \\
\hline $\begin{array}{l}\text { Sprachschicht } \\
\text { bzw. Stilebene }\end{array}$ & $\begin{array}{l}\text { Standardsprache bis } \\
\text { gehobene Sprache (viele } \\
\text { Okkurrenzen in literarischen } \\
\text { Werken) }\end{array}$ & Standard- und Umgangssprache \\
\hline Sprache & $\begin{array}{l}\text { Geschriebene und gespro- } \\
\text { chene Sprache }\end{array}$ & Viele Okkurrenzen in Gesprächen \\
\hline $\begin{array}{l}\text { Partner des StSa } \\
\text { (d. h. häufige } \\
\text { Kookkurrenzen) }\end{array}$ & $\begin{array}{l}\text {-et (puis) après?(ist übrigens } \\
\text { auch ein StSa) }\end{array}$ & $\begin{array}{l}\text { Partikeln: doch, ja } \text { sind besonders } \\
\text { häufig; } \text { nun, wohl weniger. }\end{array}$ \\
'bah'); pardi( $\approx$ 'na klar') & $\begin{array}{l}\text { Kombinationen von Partikeln sind } \\
\text { möglich. }\end{array}$ \\
\hline
\end{tabular}

Tabelle 5: Gebrauch des StSa

\subsubsection{Pragmatische Funktionen}

Das ist die Höhe drückt aus, dass etwas zu weit geht, übertrieben ist. Die Reaktion kann Staunen, Verwunderung sein, aber auch Missbilligung und sogar Empörung und dies sehr oft in einem Zwei-Schritt-Verfahren. Ein erster Grund zur Missbilligung wird angegeben, dann ein zweiter, wichtigerer, nämlich eine Art Katalysator, der die Reaktion (d. h. das ist die Höhe) herbeiführt. Im Beispiel unten ist der erste Grund zur Empörung die Tatsache, dass der Verkehrsrowdy ein gefährlicher Autofahrer ist. Der zweite Grund ist seine Frechheit.

Risikofahrt die L 531 hinab: Als der Rowdie gestoppt wurde, pöbelte er. SIEGEN-EISERFELD.

Das ist die Höhe: Ein 35-jähriger Verkehrsrowdie gefährdete am Sonntag auf der Eiserfelder Straße von Neunkirchen nach Eiserfeld auf dem Gefällestück am Waldhaus "Schränke" ein halbes Dutzend anderer Verkehrsteilnehmer und wurde, als sie ihn zur Rede stellten, auch noch ausfallend. (Rhein-Zeitung, 2006, IdS-Korpus)

Bei la belle affaire ist die pragmatische Ebene komplizierter. Die zentrale pragmatische Funktion ist das Minimieren, das Relativieren einer Aussage, einer Tatsache. Dieses Minimieren hat mehrere Varianten, die in Tabelle 6 dargestellt werden:

\begin{tabular}{|l|l|}
\hline Pragmatische Funktion & Beispiel \\
\hline $\begin{array}{l}\text { eine Aussage/ eine Tatsache } \\
\text { banalisieren } \\
(\approx \text { 'eigentlich ist es ganz normal') }\end{array}$ & $\begin{array}{l}\text { L'on me raconta sans m'étonner que Charles Quint } \\
\text { avait ramassé le pinceau du Titien: la belle affaire! } \\
\text { un prince est fait pour cela (Sartre 1964: 51) }\end{array}$ \\
\hline etw. akzeptieren, jm. zustimmen & $\begin{array}{l}\text {-Est-ce que cela ne t'embête pas trop de garder mon } \\
\text { fils ce week-end? } \\
- \text { La belle affaire! }\end{array}$ \\
\hline
\end{tabular}




\begin{tabular}{|l|l|}
\hline etw. kritisieren, in Frage stellen & $\begin{array}{l}\text { Deux cent francs! La belle affaire! C'est mon dû que } \\
\text { je veux, c'est dix mille francs (TLFi, Artikel beau) }\end{array}$ \\
\hline $\begin{array}{l}\text { eine Aussage zunichte machen, } \\
\text { zugrunde richten } \\
(\approx \text { 'es hat keinen Sinn, es ist } \\
\text { zwecklos') }\end{array}$ & $\begin{array}{l}\text { - Il a trouvé ton carnet et il l'a lu en cachette [...]: } \\
- \text { La belle affaire. Il n'a rien pu comprendre (Troyat } \\
\text { 1947: } 64)\end{array}$ \\
\hline $\begin{array}{l}\text { ablehnen und sogar } \\
\text { herausfordern }\end{array}$ & $\begin{array}{l}\text { Ils cherchent à obtenir ma main, la belle affaire! Je } \\
\text { suis riche et mon père avancera son gendre. } \\
\text { (Stendhal 1983: 330) }\end{array}$ \\
\hline
\end{tabular}

Tabelle 6: Pragmatische Funktionen des StSa

\subsubsection{Diskursive Funktionen}

Es gibt eine ganze Reihe von Kriterien, mit denen die diskursiven Funktionen und Verwendungen der StSa beschrieben werden können. Diese Kriterien bestimmen die Strukturierung der verschiedenen Belege im Wörterbuchartikel. Sie tragen dazu bei, folgende Fragen zu beantworten:

- Ist der StSa die Reaktion auf eine Aussage, auf einen Fakt, auf den Ausdruck eines Gefühls?

- Ist der StSa die Reaktion des Sprechers auf die Aussage eines anderen Sprechers oder auf seine eigene Aussage?

- Ist der StSa eine Reaktion innerhalb eines Gesprächs oder einer Erzählung? Ist es ein spontanes Gespräch? Die für das StSa-Projekt ausgewerteten Korpora bestehen aus schriftlich fixierten Texten, d. h. aus Romanen, Krimis, Essays und Zeitungsartikeln. Die Gespräche, in denen die StSa auftreten, können folglich nicht als spontane, natürliche Gespräche gelten (vgl. Brinker/Sager 2012: 14).

- Wie folgen Motiv und Reaktion aufeinander? Der StSa ist zwar eine Reaktion, aber im Gespräch steht er manchmal vor dem Motiv.

\subsection{7 Übersetzung der StSa}

Die französischen Äquivalente (Übersetzungen) von das ist die Höhe stehen in der ersten Zeile der Tabelle. Die generische Übersetzung (zweite Zeile) kann grundsätzlich in allen Kontexten gebraucht werden. In der letzten Zeile wird eine Klassifizierung der Äquivalente des StSa vorgeschlagen: Sie ermöglicht es, die verschiedenen pragmatischen Funktionen des StSa in der Zielsprache darzustellen.

\begin{tabular}{|l|l|}
\hline \multicolumn{2}{|c|}{ das ist die Höhe } \\
\hline $\begin{array}{l}\text { Französische Äquivalente } \\
\text { im Korpus }\end{array}$ & $\begin{array}{l}\text { alors, ça, quel culot/(oh, }) \quad c ̧ a \text { alors!/ça, c'est la } \\
\text { meilleure/ça dépasse les bornes/(ça/mais), c'est le/un } \\
\text { comble/c'est complet/c'est insensélc'est la fin de tout/c'est } \\
\text { la fin des haricots/c'est la totale/c'est le bouquet/c'est le }\end{array}$ \\
\hline
\end{tabular}




\begin{tabular}{|l|l|}
\hline & $\begin{array}{l}\text { pompon/c'est fort de cafél (ça,) c'est trop fortl (ça) c'est } \\
\text { incroyablelen plus/il ne manquait plus que celal on aura } \\
\text { tout vu/entendu/par exemple!/un comble! }\end{array}$ \\
\hline Generische Übersetzung & c'est le/un comble \\
\hline $\begin{array}{l}\text { Pragmatische Klassi- } \\
\text { fizierung der Äquivalente: } \\
\text { was drücken sie aus? }\end{array}$ & $\begin{array}{l}\text { - Staunen, Verwunderung: c'est fort de café/X (être) } \\
\text { exemple!/quel culot! } \\
\text { - Übertreibung: c'est le bouquet/c'est la meilleure/c'est le } \\
\text { pompon/c'est la totale/c'est complet/c'est trop fort/c'est } \\
\text { un comble/ça dépasse les bornes/il ne manquait plus que } \\
\text { cela } \\
\text { - Resignation: c'est la fin de tout/c'est la fin des haricots }\end{array}$ \\
\hline
\end{tabular}

Tabelle 7: Übersetzung der StSa

Die ersten Wörterbuchartikel sind vor kurzem in den Nouveaux Cahiers d'Allemand(Nancy) ${ }^{10}$ veröffentlicht worden: Métrich (2012), Kauffer (2012), GualbertoSchneider und Balnat (2012), Nahon-Raimondez (2012).

\section{Literatur}

Balzer, Berit/Piñel, Rosa (2010): "Vorschlag eines klassifikatorischen Modells für Routineformeln psycho-sozialer Art". In: Korhonen, Jarmo/Mieder, Wolfgang/Piirainen, Elisabeth/Piñel, Rosa (eds.) (2009): Phraseologie globa l-areal - regional. Akten der Konferenz Europhras 2008 vom 13-16.8.2008 in Helsinki. Tübingen, Narr: 269-275.

Bárdosi, Vilmos/Ettinger, Stefan/Stölting, Cécile ( $\left.{ }^{3} 2003\right)$ : Redewendungen Französisch/ Deutsch. Thematisches Wörter- und Übungsbuch. Tübingen/Basel: Francke/UTB.

Bidaud, Françoise (2002): Structures figées de la conversation. Analyse contrastive françaisitalien. Bern: Lang.

Blum, Claude (ed.) (2007): Le nouveau Littré. Paris : Garnier.

Brinker, Klaus/Sager, Sven F. (2012): Linguistische Gesprächsanalyse. Berlin: Schmidt.

Burger, Harald (1973): Idiomatik des Deutschen. Tübingen: Max Niemeyer.

Burger, Harald (1998/22003): Phraseologie. Eine Einführung am Beispiel des Deutschen. Berlin: Schmidt.

Burger, Harald/Buhofer, Annelies/Sialm, Ambros (1982): Handbuch der Phraseologie. Berlin/New-York: de Gruyter.

Burger, Harald/Dobrovol'ski, Dimitrij/Kühn, Peter/Norrick, Neal R. (eds.) (2007): Phraseologie/Phraseology. Berlin/New York: Mouton/de Gruyter. (=Handbücher zur Sprach- und Kommunikationswissenschaft 28).

Coulmas, Florian (1981): Routine im Gespräch. Zur pragmatischen Fundierung der Idiomatik. Wiesbaden: Athenaion.

Coulon-Mrosowski, Bettina (1992): 3500 locutions idiomatiques, lexique allemand-français, français-allemand. Paris: Nathan.

\footnotetext{
${ }^{10}$ Einzelheiten unter http://www.atilf.fr/spip.php?rubrique203, Stand: 19.11.2013.
} 
Digitales Wörterbuch der deutschen Sprache. http://www.dwds.de/, Stand: 31.10.2013.

Dobrovol'skij, Dimitrij (1999): "Phraseologische Wörterbücher Deutsch-Russisch und Russisch-Deutsch. Stand und Perspektiven". Germanistische Linguistik 143-144: 141-175.

Dostie, Gaétane (2004): Pragmaticalisation et marqueurs discursifs. Analyse sémantique et traitement lexicographique. Brüssel: de boeck duculot.

Dubois, Jean (ed.) (1977): Larousse de la Langue Française. Paris : Larousse.

Dudenredaktion (2002): Duden 11 - Redewendungen. Mannheim usw.: Dudenverlag.

Dudenredaktion (2003): Duden - Deutsches Universalwörterbuch. 5. Auflage. Mannheim usw.: Dudenverlag.

Dziadkiewicz Aleksandra (2007): "La traduction automatique de phraséologismes pragmatiques: quelles représentations à travers la diversité formelle et structurelle?". Corela, 5/2. http://corela.edel.univ-poitiers.fr/index.php?id=383, Stand: 03.12.2013.

Fónagy, Ivan (1997): "Figement et changements sémantiques". In: Martins-Baltar, Michel (ed.) (1997): La locution entre langue et usages. Paris, ENS Editions/Ophrys: 131-164.

Greciano, Gertrud (1995): "Fachphraseologie". In: Métrich, René/Faucher, Eugène (eds.) (1995):Rand und Band - Abgrenzung und Verknüpfung als Grundtendenzen des Deutschen. Tübingen, Narr: 183-195.

Glück, Helmut (ed.) (1993): Metzler Lexikon Sprache. Stuttgart/Weimar: Metzler.

Gspann, Lucien (1971): Gallicismes et germanismes à gogo. Paris: Didier.

Gualberto-Schneider, Antje/Balnat,Vincent (2012): "Petit dictionnaire permanent des 'actes de langages stéréotypés'(ALS). Microstructure de 'na warte!"'. Nouveaux Cahiers d'allemand 3: 269-284.

Gualberto-Schneider, Antje/Kauffer, Maurice/Nahon-Raimondez, Anne-Marie (im Druck): "Réflexions sur le figement des 'phraséologismes pragmatiques' en français et en allemand". In: Gautier Laurent u.a. (eds.) (im Druck): Les phénomènes de figement en linguistique. München: Meidenbauer.

Gülich, Elisabeth/Henke, Käthe (1979/1980): "Sprachliche Routine in der Alltagskommunikation". Die neueren Sprachen 78: 513-530 \& 79: 2-33.

Higi-Wydler, Melanie (1989): Zur Übersetzung von Idiomen - Eine Beschreibung und Klassifizierung deutscher Idiome und ihrer französischen Übersetzungen. Bern: Lang.

Hullmann, Wolfgang (1996): Lexique français-allemand des clichés de presse et expressions du quotidien. Paris: Ellipses.

Hyvärinen, Irma (2011): "Zur Abgrenzung und Typologie pragmatischer Phraseologismen." In: Hyvärinen, Irma/Liimatainen, Annikki (eds.) (2011): Beiträge zur pragmatischen Phraseologie. Frankfurt a. M., Peter Lang: 9-43. (=Finnische Beiträge zur Germanistik 25).

Kauffer, Maurice (2007): "Le projet Lexitec - Comment constituer un dictionnaire électronique bilingue des expressions idiomatiques?". Nouveaux Cahiers d'allemand 1: 23-49.

Kauffer, Maurice (2012): "Petit dictionnaire permanent des 'actes de langages stéréotypés'. Microstructure de 'das ist die Höhe'". Nouveaux Cahiers d'allemand2: 129-145.

Korhonen, Jarmo (2007): "Probleme der kontrastiven Phraseologie". In: Burger u. a. (eds.) (2007): 574-589.

Kühn, Peter (1983): "Pragmatische und lexikographische Beschreibung phraseologischer Einheiten: Phraseologismen und Routineformeln". Germanistische Linguistik 1-3: 141-175. Lamiroy, Béatrice (2010): Les expressions figées de la francophonie. Paris: Ophrys. 
Lubensky, Sophia/Mac Shane, Marjorie (2007): "Bilingual phraseological dictionaries". In: Burger u. a. (eds.) (2007): 919-928.

Lüger, Heinz-Helmut (1999): Satzwertige Phraseologismen. Eine pragmalinguistische Untersuchung. Wien: Praesens.

Lüger, Heinz-Helmut (2007): "Pragmatische Phraseme: Routineformeln". In: Burger u. a. (eds.) (2007): 444-459.

Martins-Baltar, Michel (2000): "Les énoncés usuels et le modèle motif $\leftrightarrow$ réaction". In: Greciano, Gertrud (ed.) (2000): Micro- et macrolexèmes et leur figement discursif. Louvain/Paris, Peeters: 93-106.

Martins-Baltar, Michel (2006): "Comment constituer une nomenclature en pragmatographie?" In: Szende, Thomas (ed.) (2006): Le français dans les dictionnaires bilingues. Paris, Champion: 193-203.

Mel'čuk, Igor (2008): "Phraséologie dans la langue et dans le dictionnaire" In: Campà, Àngels/Baqué, Lorraine (eds.) (2008): Repères \& Applications (VI), XXIVe Journées Pédagogiques sur l'Enseignement du Français en Espagne, Barcelone 3-5 septembre 2007: 187-200.

Métrich, René(2011): "Zur Konzeption eines bilingualen Wörterbuchs der 'stereotypen Sprechakte' am Beispiel von tu parles!". In: Schäfer, Patrick/Schowalter, Christine (eds.) (2011): In mediam linguam. Mediensprache - Redewendungen - Sprachvermittlung. Landau, Verlag Empirische Pädagogik: 331-348.

Métrich, René (2012): "Microstructure de 'tu parles"'. Nouveaux Cahiers d'allemand1: 3-17. Müller, Peter O./Kunkel-Razum, Kathrin (2007): "Phraseographie des Deutschen". In: Burger u. a. (eds.) (2007): 939-949.

Nahon-Raimondez, Anne-Marie (2012): "Petit dictionnaire permanent des 'actes de langages stéréotypés' (ALS). Microstructure de 'n'empêche"'. Nouveaux Cahiers d'allemand 4: 367381.

Projekt Gutenberg-DE (ed.) ( $\left.{ }^{12} 2011\right)$ : Gutenberg-De. Hamburg: Hille \& Partner.

Rey, Alain/Chantreau, Sophie (1997): Dictionnaire d'expressions et locutions. Paris: Le Robert. Robert, Paul (ed.) (2001): Le Petit Robert. Paris : Le Robert.

Schemann, Hans (1993): Deutsche Idiomatik - Die deutschen Redewendungen im Kontext. Stuttgart/Dresden: Klett.

Schneider, Franz (1989): Comment décrire les actes de langage? Tübingen: Niemeyer.

Schmale, Günter (2011). "Was ist in der Sprache 'vorgeformt'?. Überlegungen zu einer erweiterten Definition sprachlicher Präformiertheit". In: Schäfer, Patrick/Schowalter, Christine (eds.) (2011): In mediam linguam. Mediensprache - Redewendungen Sprachvermittlung. Landau: VEP, 177-190.

Schulz, Dora/Griesbach, Heinz (1975): 1000 idiomatische Redensarten. Berlin usw.: Langenscheidt/Hachette.

Stein, Stephan (1995): Formelhafte Sprache. Untersuchungen zu ihren pragmatischen und kognitiven Funktionen im gegenwärtigen Deutsch. Frankfurt a. M.: Lang.

Stein, Stephan (2004): "Formelhaftigkeit und Routinen in mündlicher Kommunikation". In: Steyer, Kathrin (ed.) (2004): Wortverbindungen - mehr und weniger fest. Berlin/New-York, de Gruyter: 262-288.

Trésor de la langue français informatisé, http://atilf.atilf.fr, Stand: 31.10.2013. 
Werny, Paul/Snyckers, Alexandre (1982): Dictionnaire des locutions français-allemand. Paris: Larousse.

Zifonun, Gisela/Hoffmann, Ludger/Strecker, Bruno (1997): Grammatik der deutschen Sprache. 3 Bände. Berlin/New York: de Gruyter.

Zimmer, Rudolf (1990): Äquivalenzen zwischen Französisch und Deutsch. Theorie - Korpus -Indizes. Ein Kontextwörterbuch. Tübingen: Niemeyer.

\section{Quellen der Belege}

Buron, Nicole de (1996): T'as tout pour être heureuse. Paris: Flammarion.

Chandernagor, Françoise (1981): L'Allée du Roi. Paris: Julliard.

Cohen, Albert (1930): Solal. Paris: Gallimard.

Dorner, Françoise (2008): Die letzte Liebe des Monsieur Armand (übersetzt von C. Gersch). Zürich: Diogenes Taschenbuch.

Ikor, Roger (1955): Les Fils d'Avrom. Paris: Albin Michel.

Queneau, Raymond (1967): Zazie dans le métro. Paris: Gallimard.

Sartre, Jean-Paul (1943): Les Mouches. Paris: Gallimard.

Sartre, Jean-Paul (1964): Les Mots. Paris: Gallimard.

Stendhal (1983): Le rouge et le noir. Paris: Librairie générale Française.

Troyat, Henri (1947): Tant que la terre durera. Paris: La Table Ronde. 